\title{
Editorial: Special Issue on Weather and Climate around Mountains
}

The International Conference on Alpine Meteorology (ICAM) had its 29th realisation in the town of Chambéry in the French department of Haute Savoie from 4-8 June 2007, jointly organised by Météo France and the Laboratoire d'Aérologie of CNRS and University of Toulouse. Some 230 participants from 22 countries on 3 continents reconfirmed in a wider and more general sense the final words of the opening speech in Italian quoted above, which inaugurated the first conference nearly 57 years earlier. Once more the researchers demonstrated through their science of meteorology and its numerous applications that mountain ranges, such as the Alps, contribute to uniting people rather than dividing them.

Following a brief opening ceremony containing an address from the World Meteorological Organization, the conference contained 79 short oral presentations grouped into 18 sessions and 141 poster displays, which were discussed intensely during three extended poster sessions. Authors, titles and extended abstracts can still be viewed online at www.cnrm.meteo.fr/icam2007/ html/PROCEEDINGS/.

Following the tradition introduced at the 27th ICAM in Brig, Switzerland (2003) and maintained at the 28th ICAM in Zadar, Croatia $(2005)^{2}$, the presenters were invited to extend their presentations into concise articles for a common publication; this time in the research journal Meteorology and Atmospheric Physics. No less than 34 manuscripts were received before the end of 2007 and underwent the regular review

\footnotetext{
1 cf. Bossolasco M (1950) Atti del primo convegno internazionale di meteorologia alpina. Pure Appl Geophys 17: 81-7

2 cf. Meteorol Z 13 (parts of issues 1-3, 2004) and Meteorol Z 15: issue 2, 2006
}

process. In this issue 23 accepted papers are assembled following a prelude that highlights the remarkable series of ICAM, which is now widely accepted as the European communication forum for mountain meteorology with strong links to the Mountain Meteorology Conferences organised by the American Meteorological Society. The order of papers loosely follows the themes selected for the conference sessions. Four groups are distinguished:

"Precipitation: simulated and observed" contains seven articles. Orr and Bechtold demonstrate how warm season rainfall near major mountain ranges can be predicted more accurately in the ECMWF global model. Godart et al. address in a regional modelling study subtleties of banded convection and rainfall regimes at the edge of the Massif Central in France. Davolio et al. revisit a flash flood case in the same region and demonstrate in a modelling study how the underlying terrain can trigger long lived convection. Likewise Bresson et al. apply a research-type forecasting system with realistic orography and idealized initial conditions to elucidate the main ingredients for high precipitation rates in southern France. Brynjólfsson and Ólafsson document the large spatial gradients of precipitation at mountain slopes in Northern Iceland. The regional modelling study by Rontu et al. reveals the importance of surface energy balances over a glacier for mesoscale predictions. Mariani et al. apply novel verifications techniques for a multi-model intercomparison of strong precipitation events in the Alpine area.

The next six papers address various aspects of "Predictability and Applications". Trentmann et al. use a series of multi-model simulations to 
evaluate their skill for a convective case at low mountains of Vosges and Black Forest. Argence et al. apply for the same region a non-hydrostatic modelling system for a sample of ten cases and put the emphasis on the role of different large scale forcing data. Vincedon et al. undertake the step towards coupled hydro-meteorological simulations for flash-flood warnings by assessing the quality of forecast precipitation fields at the flanks of the Massif Central. Pradier-Vabre et al. describe a trial application of such convective-scale numerical weather prediction tools in a flight simulator for enhanced safety in aviation. Schneider and Steinacker concentrate on measurements in a study of how to refine precipitation fields from radar observations. Finally, Chan demonstrates how atmospheric turbulence in complex terrain can be inferred from a blend of model results with remote sensing data.

Seven articles cover the wide topic "Dry flows: gusts, waves and air quality". Horvath and IvančanPicek link Mediterranean lee cyclones to underlying mountain ranges and mesoscale potential vorticity anomalies. Ágústsson and Ólafsson investigate wind gusts in Iceland as important features of a much smaller scale. Chemel et al. perform a numerical study of the oscillatory behaviour of katabatic winds in an alpine valley and the associated generation of gravity waves. Yao and Zhong document temperature inversions in the small basin of a meteoritic crater and relate it to the ambient atmosphere. Schicker and Seibert concentrate on a large Alpine valley and the simulation of a winter smog episode. Harnisch et al. investigate the aerosol distributions in the Inn valley during winter. Like both previous studies, de Franceschi and Zardi deal with high pollution measurements taken during the European-Union project ALPNAP, in their case from the Adige valley south of the main Alpine crest.

The final group "Climate: techniques and time series" comprises three articles. Salameh et al. concentrate on statistical downscaling methods from climate-scenario-simulations for southern France with distinct variations in regional topography. Andrighetti et al. introduce a homogenized temperature series for Verona at the southern Alpine flank, which covers 240 years. And finally, Aquotta et al. investigate various time series at several stations in the neighbouring Piedmont region.

Although each paper contains its quite specific findings, which are relevant for a larger or smaller part of the community, a synoptic view over the entire issue reveals additional information. Current mountain meteorological studies have a wide scope encompassing weather and climate, complex observations and numerical simulation, basic studies and user oriented applications to name but a few pairs of categories. Of the scientific papers, 13 contributions stem from oral presentations and 10 originate from posters. This demonstrates that at ICAM posters are indeed an important class of presentation - often very interactive - of equal importance and status to the oral presentations. The considerable educational aspect of international conferences like ICAM becomes evident as nearly half of the lead authors presented parts of their theses or immediate post-doctoral work.

The international team of guest-editors acknowledges with gratitude the pronounced cooperative spirit between many individuals without which this collection of papers could not have been assembled; specifically: the co-authoring teams for timely production of manuscripts and revisions, all reviewers for provision of constructive criticism, the editorial team around Reinhold Steinacker and Barbara Chimani for dedicated support and much personal interest in mountain meteorology, and the staff of Springer Wien New York - Wolfgang Dollhäubl in particular.

We hope that all articles will enjoy a broad and interested readership, furthering common scientific understanding and enhancing unity between people, even when mountains appear to stand in the way.

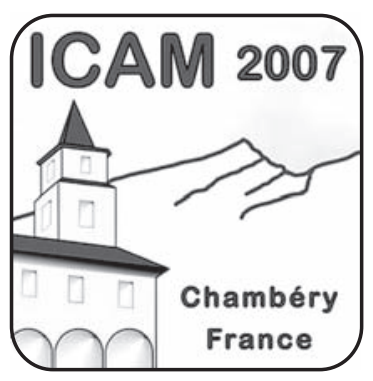

Evelyne Richard (Toulouse) Andrea Buzzi (Bologna) Stephen Mobbs (Leeds) Hans Volkert (Oberpfaffenhofen) Mark Žagar (Ljubljana) 